\title{
A Structural Model of Stress, Motivation, and Academic Performance in Medical Students
}

\author{
Jangho Park', Seockhoon Chung ${ }^{1} \bowtie$, Hoyoung $\mathrm{An}^{2}$, Seungjin Park ${ }^{1}$, \\ Chul Lee ${ }^{1}$, Seong Yoon Kim', Jae-Dam Lee ${ }^{3}$ and Ki-Soo Kim ${ }^{4}$ \\ 'Department of Psychiatry, University of Ulsan College of Medicine, Asan Medical Center, Seoul, Korea \\ ${ }^{3}$ Department of Medical Humanities and Social Sciences, University of Ulsan College of Medicine, Asan Medical Center, Seoul, Korea \\ ${ }^{4}$ Department of Pediatrics, University of Ulsan College of Medicine, Asan Medical Center, Seoul, Korea \\ ${ }^{2}$ Jeju Mental Sanatorium, Jeju, Korea
}

Objective The purpose of the present study was 1) to identify factors that may influence academic stress in medical students and 2) to investigate the causal relationships among these variables with path analysis.

Methods One hundred sixty medical students participated in the present study. Psychological parameters were assessed with the Medical Stress Scale, Minnesota Multiphasic Personality Inventory, Hamilton Depression Scale, Beck Depression Inventory, and Academic Motivation Scale. Linear regression and path analysis were used to examine the relationships among variables.

Results Significant correlations were noted between several factors and Medical Stress scores. Specifically, Hamilton Depression Scale scores $(\beta=0.26, p=0.03)$ and amotivation $(\beta=0.20, p=0.01)$ and extrinsically identified regulation $(\beta=0.27, p<0.01)$ response categories on the Academic Motivation Scale had independent and significant influences on Medical Stress Scale scores. A path analysis model indicated that stress, motivation, and academic performance formed a triangular feedback loop. Moreover, depression was associated with both stress and motivation, and personality was associated with motivation.

Conclusion The triangular feedback-loop structure in the present study indicated that actions that promote motivation benefit from interventions against stress and depression. Moreover, stress management increases motivation in students. Therefore, strategies designed to reduce academic pressures in medical students should consider these factors. Additional studies should focus on the relationship between motivation and depression.

Psychiatry Investig 2012;9:143-149

Key Words Academic performance, Depression, Medical school, Motivation, Stress.

\section{INTRODUCTION}

Medical education is associated with high stress. ${ }^{1,2}$ Indeed, approximately $10 \%$ of medical students suffer from major depressive disorder, and $6 \%$ have a history of suicidal ideation. ${ }^{3}$ These reports have raised concerns in many medical education institutions, and recent reports have focused on this topic. ${ }^{1,3-6} \mathrm{~A}$ greater understanding of the factors underlying academic stress may lead to improved stress-management strategies.

Received: August 29, 2011 Revised: December 27, 2011

Accepted: January 30, 2012 Available online: April 2, 2012

$\triangle$ Correspondence: Seockhoon Chung, $\mathrm{MD}, \mathrm{PhD}$

Department of Psychiatry, University of Ulsan College of Medicine, Asan Medical Center, 88 Olympic-ro 43-gil, Songpa-gu, Seoul 138-736, Korea Tel: +82-2-3010-3411, Fax: +82-2-485-8381, E-mail: schung@amc.seoul.kr

(c) This is an Open Access article distributed under the terms of the Creative Commons Attribution Non-Commercial License (http://creativecommons.org/licenses/bync/3.0) which permits unrestricted non-commercial use, distribution, and reproduction in any medium, provided the original work is properly cited.
Various factors contribute to academic stress in medical students. Previous studies have suggested that stress-related factors may be interlinked, forming an intricate psychological structure that may precipitate and perpetuate academic stress. For instance, academic stress is associated with academic performance, ${ }^{7}$ personality, ${ }^{8}$ academic motivation, ${ }^{9}$ and depression. ${ }^{10}$ Personality has been linked with both academic performance ${ }^{11,12}$ and motivation. ${ }^{13}$ Moreover, previous studies have reported an association between academic performance and motivation. ${ }^{14}$ All of these factors may therefore be included in the structure, and investigation of this structure may provide valuable information about academic stress.

Previous studies have focused on academic motivation much less than on other factors. One of the most widely acknowledged theories on this topic is the self-determination theory. ${ }^{9}$ It suggests that academic motivation can be divided into intrinsic motivation, extrinsic motivation, and amotivation based on the in- 
fluence of personal needs and drive and their interaction with external pressures. ${ }^{16}$ Intrinsic motivation is defined as drive from personal needs and satisfaction and is further split into three subcategories based on the type of satisfaction experienced during the activity: intrinsic motivation to know, intrinsic motivation to accomplish, and intrinsic motivation to experience stimulation. Intrinsic motivation to know refers to motivation based on the enjoyment of learning new things. Intrinsic motivation to accomplish refers to the motive to accomplish or to create new things. Intrinsic motivation to experience stimulation refers to motivation to achieve sensory stimulation while performing an activity. Extrinsic motivation is based on a drive coming from environmental factors or a sense of obligation and is divided into four subtypes: external regulation, introjected regulation, identified regulation, and integrated regulation. External regulation is motivation deriving from external influences or rewards. Introjected regulation is motivation from the environment that generates internal reward contingencies and includes a slightly higher level of self-determination compared with external regulation. Identified regulation is motivation that has been incorporated into personal attributes and external factors and involves a higher level of self-determination than introjected regulation does. Integrated regulation is motivation that is slightly different from internal motivation. It involves the highest level of self-determination among the external motivation subtypes. This type of motivation is externally regulated, but the individual also enjoys the activity in itself. In contrast, amotivation is the absence of drive. Intrinsic motivation, extrinsic motivation, and amotivation can be arranged along a motivational continuum based on the amount of self-determination. Moreover, the four subcategories of extrinsic motivation can also be arranged along a continuum based on the same principle (Figure 1$){ }^{9}$

The present study examined the relationships between various factors associated with academic stress in medical students, including motivation, personality, academic performance, and depression. The causal relationships among these factors were investigated via path analysis. Our findings may form the basis for management of student mental health in medical schools.

\section{METHODS}

\section{Participants}

The present study was based on the results of a survey that focused on evaluating and promoting mental health in medical students. The survey was conducted in November 2009 at the University of Ulsan College of Medicine in Seoul, Korea. Korean students may apply to medical school, which consists of 4 years of training, after receiving an undergraduate degree. They may also apply immediately after finishing high school.

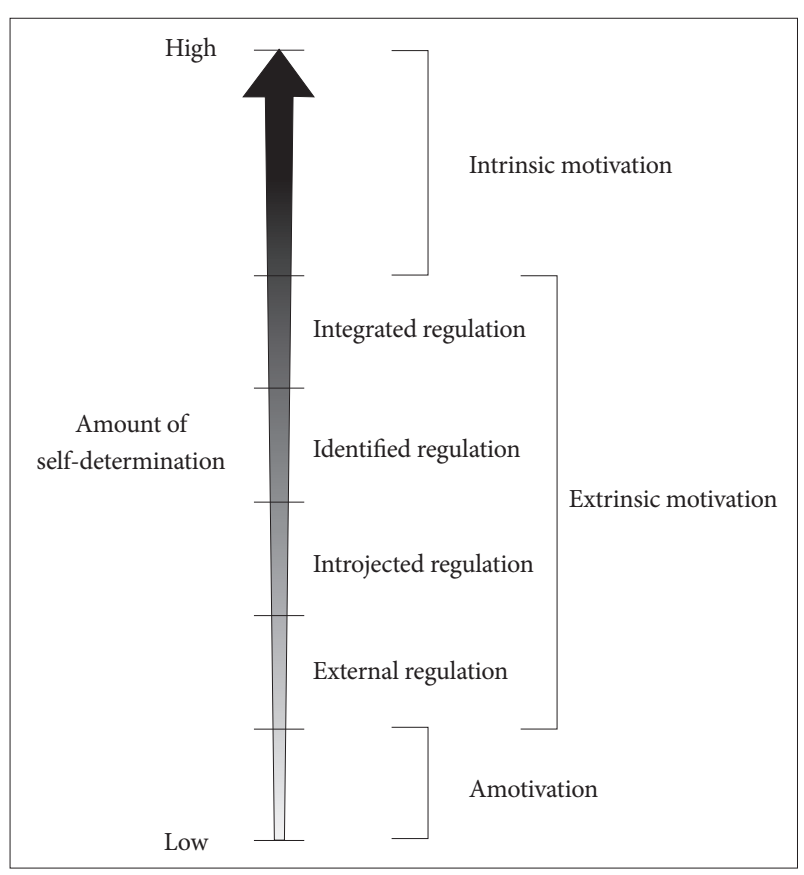

Figure 1. The structure of motivation. Based on Deci and Ryan's selfdetermination theory.

In this case, students take a 2-year pre-medicine curriculum prior to 4 years of medical school. The present study was conducted at one of the latter institutions. The survey was organized by school administrators and was taken by 160 students who were entering their third year of education (i.e., first year of medical school). The curriculum of the students included basic science courses (i.e., anatomy and histology) during the second year of pre-medicine coursework. During the first 2 years of medical school, the students are enrolled in extended basic science/clinical coursework (e.g., cardiology and reproductive medicine), and the last 2 years consist of clinical ward training. The aims of the survey were explained in detail to all of the students, and questionnaires were handed out. The students were given ample time to complete the survey. During the 4week period after the surveys were completed, the students visited the psychiatry outpatient clinic and received individual assessments conducted by a psychiatrist. The psychiatric evaluation included the Structured Clinical Interview for DSM-IV (SCID-IV) and the Mini-International Neuropsychiatric Interview (MINI). The interviews were conducted according to the principles of SCID-IV and MINI, although due to time constraints, the tests were not completed in full. Students who were determined to be at high risk for mental health problems were asked to participate in a second interview. All of the students participated in at least one interview. None of the students in the present study was diagnosed with a significant mental health problem. Informed consent was provided by all of the students, and all of them were included in the final analyses. 
The Institutional Review Board at the Asan Medical Center approved the present study.

\section{Measures}

The following questionnaires were used in the present study: the Medical Stress Scale (MSS), ${ }^{15}$ the Minnesota Multiphasic Personality Inventory (MMPI-2), ${ }^{17}$ the Hamilton Depression scale (HAM-D-17), ${ }^{18}$ the Beck Depression Inventory (BDI-II), ${ }^{19}$ and the Academic Motivation Scale (AMS). ${ }^{15}$ After participants had completed the four self-report questionnaires (MSS, MMPI-2, BDI-II, and AMS), the psychiatrists examined all participants, and depressive symptoms were further assessed with the HAM-D-17.

The MSS is an 11-item questionnaire designed to assess the amount of stress medical students experience during medical school. ${ }^{8}$ It focuses on five areas including school curriculum and environment, personal competence/endurance, social/recreational life, financial situation, and future aspirations. Each item is scored on a 5-point Likert scale ranging from 'strongly disagree' to 'strongly agree.' Higher scores represent higher levels of stress. The reliability and content validity of this assessment are high. ${ }^{8} \mathrm{~A}$ validated Korean version was used in the present study. The original test was modified to reflect cultural differences and consisted of 9-items in three areas: school curriculum and environment, personal competence/endurance, and financial situation. ${ }^{15}$ Participants with MSS scores $\geq 28$ were categorized as 'stressed' $(n=56)$, and the others were considered 'non-stressed' $(n=104)$. Each section of the Korean version of the MSS ${ }^{15}$ consists of nine items scored on a 5-point Likert scale. A 3-point answer ('neither agree nor disagree') for each item would result in a total score of 27 points. Hence, a score of 28 was set as the cutoff point for the present study. This dichotomized categorization was used in the initial analysis; hereafter, raw scores were used in all analyses.

The AMS is a 28-item questionnaire developed to describe academic motivation ${ }^{16}$ based on self-determination theory. ${ }^{9}$ It assesses three subcategories of intrinsic motivation (i.e., intrinsic motivation to know, intrinsic motivation to accomplish things, and intrinsic motivation to experience stimulation), three subcategories of extrinsic motivation (i.e., extrinsic external motivation, external introjected motivation, and extrinsic identified motivation), and amotivation. Higher scores in each subcategory reflect higher levels of motivation based on that subcategory. A validated Korean version of the AMS was used. ${ }^{15}$

The MMPI-2 is an instrument used to identify personality structures and psychopathology. ${ }^{17}$ It consists of 567 items that are combined in a variety of ways to yield different scales. The clinical scales, which focus on psychopathology, are the most widely used. Due to our study population, scores above 65 points on the clinical scale were considered high, and the number of high scores was used for analysis. ${ }^{20}$

The HAM-D- $17^{18}$ and BDI- $\mathrm{II}^{21}$ are questionnaires that quantify depression. The 17-item HAM-D-17 is clinician based and is ideal for determining somatic symptoms. The 21-item BDI-II is a self-report inventory is focused on subjective feelings. Both measures were used for improved accuracy. Validated Korean versions were used in the present study ${ }^{19,22}$

Academic performance was assessed with school grades, which were provided by the university with consent. Gradepoint averages (GPAs; $\mathrm{A}^{+}=4.5 ; \mathrm{A}^{0}=4.0 ; \mathrm{B}^{+}=3.5 ; \mathrm{B}^{0}=3.0 ; \mathrm{C}^{+}=2.5$; $\mathrm{C}^{0}=2.0 ; \mathrm{D}^{+}=1.5 ; \mathrm{D}^{0}=1.0, \mathrm{~F}=0$ ) during the previous semester were used in the analysis.

\section{Analysis}

Chi-square analysis and Student's t-test were used to compare the demographic and clinical characteristics of stressed and non-stressed participants based on the dichotomous categorization described above. All analyses were two-tailed. Pearson's correlation was used to identify the association between various factors and stress. A linear regression model was constructed to determine the independent contribution of each factor to academic stress. Path analysis was used to estimate the causal relationship among stress, motivation, academic performance, depression, and personality. The Statistical Package for Social Sciences (SPSS) version 15.0 was used for all statistical analyses, and AMOS version 16.0 was used for the path analysis.

\section{RESULTS}

\section{Clinical characteristics of stressed and non-stressed students}

Stressed students were significantly younger compared with non-stressed students ( $21.5 \pm 1.6$ vs. $22.1 \pm 1.7$ years, $\mathrm{p}=0.03$ ). Moreover, stressed students had lower GPAs ( $3.1 \pm 0.7$ vs. $3.4 \pm$ $0.6, \mathrm{p}<0.01)$ and higher scores on the depression scales compared with non-stressed students (HAM-D-17: 8.6 \pm 6.0 vs. $4.0 \pm 3.9, \mathrm{p}<0.01$; BDI-II: $7.2 \pm 6.1$ vs. $3.3 \pm 3.7, \mathrm{p}<0.01$ ), although the mean scores were within the subclinical or normal range. Among the subcategories of the AMS, stressed students showed higher scores in amotivation (8.4 \pm 2.6 vs. $6.6 \pm 2.6, \mathrm{p}<0.01)$, intrinsic motivation to accomplish things ( $13.3 \pm 2.2$ vs. $14.2 \pm$ $2.5, \mathrm{p}=0.02)$, and extrinsic identified regulation ( $15.4 \pm 2.8$ vs. $14.0 \pm 2.8, \mathrm{p}<0.01$ ) (Table 1). No trends were noted in the distribution of MMPI-2 scales.

Separate analysis via analysis of variance (ANOVA) and posthoc analysis using the Tukey and Bonferroni methods revealed that students in their first year of medical school displayed significantly higher levels of stress compared with students in other years ( 27.0 vs. 25.5 for second-year pre-medical students, 24.1 for second-year medical students, and 22.2 for third-year medi- 
Table 1. Clinical characteristics of stressed and non-stressed

\begin{tabular}{|c|c|c|c|}
\hline \multirow{2}{*}{ Variables } & Non-stressed $(\mathrm{N}=104)$ & Stressed $(\mathrm{N}=56)$ & \multirow{2}{*}{$\mathrm{p}$} \\
\hline & $\mathrm{N}(\%)$ & $\mathrm{N}(\%)$ & \\
\hline Female gender & $28(26.7)$ & $16(28.6)$ & 0.80 \\
\hline No. of MMPI-2 clinical scales with T score $>65$ & & & $<0.01$ \\
\hline 0 & $100(95.2)$ & $45(80.4)$ & \\
\hline \multirow[t]{2}{*}{$>1$} & $5(4.8)$ & $11(19.6)$ & \\
\hline & Mean \pm SD & Mean \pm SD & \\
\hline Age (years) & $22.1 \pm 1.7$ & $21.5 \pm 1.6$ & 0.03 \\
\hline School year & $3.7 \pm 1.1$ & $3.2 \pm 1.0$ & $<0.01$ \\
\hline Grades & $3.4 \pm 0.6$ & $3.1 \pm 0.7$ & $<0.01$ \\
\hline Academic stress scale & $21.3 \pm 4.3$ & $31.2 \pm 2.8$ & $<0.01$ \\
\hline HAM-D-17 & $4.0 \pm 3.9$ & $8.6 \pm 6.0$ & $<0.01$ \\
\hline BDI-II & $3.3 \pm 3.7$ & $7.2 \pm 6.1$ & $<0.01$ \\
\hline \multicolumn{4}{|l|}{ Academic motivation scale } \\
\hline Amotivation & $6.6 \pm 2.6$ & $8.4 \pm 2.6$ & $<0.01$ \\
\hline Intrinsic motivation to know & $14.7 \pm 4.9$ & $13.4 \pm 2.1$ & 0.06 \\
\hline Intrinsic motivation to accomplish things & $14.2 \pm 2.5$ & $13.3 \pm 2.2$ & 0.02 \\
\hline Intrinsic motivation to experience stimulation & $12.5 \pm 2.6$ & $12.3 \pm 2.5$ & 0.59 \\
\hline Extrinsic external motivation & $16.2 \pm 1.9$ & $16.0 \pm 1.8$ & 0.57 \\
\hline External introjected motivation & $10.9 \pm 2.7$ & $11.5 \pm 2.8$ & 0.22 \\
\hline Extrinsic identified regulation & $14.0 \pm 2.8$ & $15.4 \pm 2.8$ & $<0.01$ \\
\hline
\end{tabular}

'Stressed' and 'Non-stressed' participants had Medical Stress Scale scores $\geq 28$ and $<28$, respectively. 'School Year' was re-coded as follows; the second year of pre-medicine as 2, the first year of medicine as 3, the second year of medicine as 4, and the third year of medicine as 5 . For 'Grades', grade-point averages $\left(\mathrm{A}^{+}=4.5 ; \mathrm{A}^{0}=4.0 ; \mathrm{B}^{+}=3.5 ; \mathrm{B}^{0}=3.0 ; \mathrm{C}^{+}=2.5 ; \mathrm{C}^{0}=2.0 ; \mathrm{D}^{+}=1.5 ; \mathrm{D}^{0}=1.0, \mathrm{~F}=0\right)$ of the previous semester were used. MMPI-2: Minnesota Multiphasic Personality Inventory, HAM-D-17: Hamilton Depression Scale, BDI-II: Beck's Depression Inventory

cal students; $\mathrm{p}<0.01$ for both post-hoc tests).

\section{Associations between demographic and psychological characteristics and stress}

The MSS scores were positively correlated with high MMPI2 scores $(\mathrm{r}=0.27, \mathrm{p}<0.01)$ and depression scale scores $(\mathrm{r}=0.45$, $\mathrm{p}<0.01$ for both) and negatively correlated with GPA ( $r=-0.29$, $\mathrm{p}<0.01)$. Five of the AMS subcategories were significantly correlated with MSS scores, including amotivation ( $\mathrm{r}=0.39, \mathrm{p}<0.01)$, intrinsic motivation to know ( $\mathrm{r}=-0.20, \mathrm{p}=0.01)$, intrinsic motivation to accomplish things $(\mathrm{r}=-0.20, \mathrm{p}=0.01)$, extrinsic external motivation $(\mathrm{r}=-0.16, \mathrm{p}=0.04)$, and extrinsic identified regulation $(\mathrm{r}=$ $0.18, \mathrm{p}=0.02$ ). Amotivation and extrinsic identified regulation were positively correlated, whereas the other three subcategories were negatively correlated with MSS scores. The depression scale scores showed the strongest correlation (Table 2).

\section{Path analysis}

The AMS and MSS results represented corresponding variables in our model. The number of high MMPI-2 scales was used to represent personality. All subcategories of the AMS, in-
Table 2. Correlation of demographic and psychological characteristics with stress

\begin{tabular}{|c|c|c|}
\hline Variables & Pearson's $\mathrm{r}$ & $\mathrm{p}$ \\
\hline Age & -0.15 & 0.06 \\
\hline Grades & -0.29 & $<0.01$ \\
\hline No. of MMPI-2 clinical scales with T score $>65$ & 0.27 & $<0.01$ \\
\hline HAM-D-17 & 0.45 & $<0.01$ \\
\hline BDI-II & 0.45 & $<0.01$ \\
\hline \multicolumn{3}{|l|}{ Academic motivation scale } \\
\hline Amotivation & 0.39 & $<0.01$ \\
\hline Intrinsic motivation to know & -0.20 & 0.01 \\
\hline Intrinsic motivation to accomplish things & -0.20 & 0.01 \\
\hline Intrinsic motivation to experience stimulation & -0.09 & 0.26 \\
\hline Extrinsic external motivation & -0.16 & 0.04 \\
\hline Extrinsic introjected motivation & 0.03 & 0.69 \\
\hline Extrinsic identified regulation & 0.18 & 0.02 \\
\hline \multicolumn{3}{|c|}{$\begin{array}{l}\text { For 'Grades', grade-point averages }\left(\mathrm{A}^{+}=4.5 ; \mathrm{A}^{0}=4.0 ; \mathrm{B}^{+}=3.5 ; \mathrm{B}^{0}=3.0 \text {; }\right. \\
\left.\mathrm{C}^{+}=2.5 ; \mathrm{C}^{0}=2.0 ; \mathrm{D}^{+}=1.5 ; \mathrm{D}^{0}=1.0, \mathrm{~F}=0\right) \text { of the previous semester were } \\
\text { used. MMPI-2: Minnesota Multiphasic Personality Inventory, HAM- } \\
\text { D-17: Hamilton Depression Scale, BDI-II: Beck's Depression Inven- } \\
\text { tory }\end{array}$} \\
\hline
\end{tabular}


cluding amotivation, were entered as indicators of the unobserved variable motivation and were arranged as covariates of each other and of the observed variable personality. The HAMD-17 and BDI-II results were arranged as indicators of the unobserved variable depression and were arranged as covariates of each other, the observed variable of personality, and the AMS subcategory of amotivation.

Previous studies have used the chi-square value, Comparative Fit Index (CFI), Normed Fit Index (NFI), and Root Mean Square Error of Approximation (RMSEA). ${ }^{23}$ The chi-square value indicates the discrepancy between the study sample and fitted covariance matrices. An insignificant chi-square value at the 0.05 level is indicative of a good model fit. The CFI compares the existing model fit with a null model, which assumes that the indicator variables and latent variables in the model are uncorrelated. A CFI value above 0.9 , which indicates that the given model can reproduce over $90 \%$ of the covariation in the data, is usually accepted as definitive. The NFI reflects the proportion by which the study model improves fit compared with the null model. Recent evidence suggests that the cutoff value for good fit should be above 0.95 . The RMSEA reflects the discrepancy per degree of freedom, and along with CFI, is among the fit indexes least affected by sample size. Although some controversy exists, a value below 0.08 or 0.05 is usually deemed adequate. ${ }^{23}$ The indices for our model satisfied all four criteria $\left(\chi^{2}=\right.$ 20.28, df=20, p=0.44; CFI=1.00; NFI=0.97; RMSEA <0.01). Among the AMS subcategories, external identified regulation had the strongest association with overall motivation, followed by extrinsic external motivation and amotivation. Motivation had a positive association with grades $(\beta=1.00)$, which in turn had a significant and negative association with stress $(\beta=-0.81$, $\mathrm{p}<0.01)$. Stress had a significant and positive association with motivation $(\beta=1.08)$, thus creating a triangular feedback loop. Depression had a positive association with stress $(\beta=0.34)$ and a negative association with motivation $(\beta=-0.75)$. Personality had a negative association with motivation. Motivation and depression were unobserved variables; therefore the statistical significance of the associations between these and other variables were not reported (Figure 2). As the data used in our study were cross-sectional, the various associations implied by our path analysis model are hypothetical.

\section{DISCUSSION}

Medical students who were under stress displayed several differences compared with students who were not stressed, and several of the factors were significantly correlated with stress levels. The results of the depression scales as well as the scores for amotivation and extrinsic identified regulation were independently associated with MSS results. The path analysis model re-

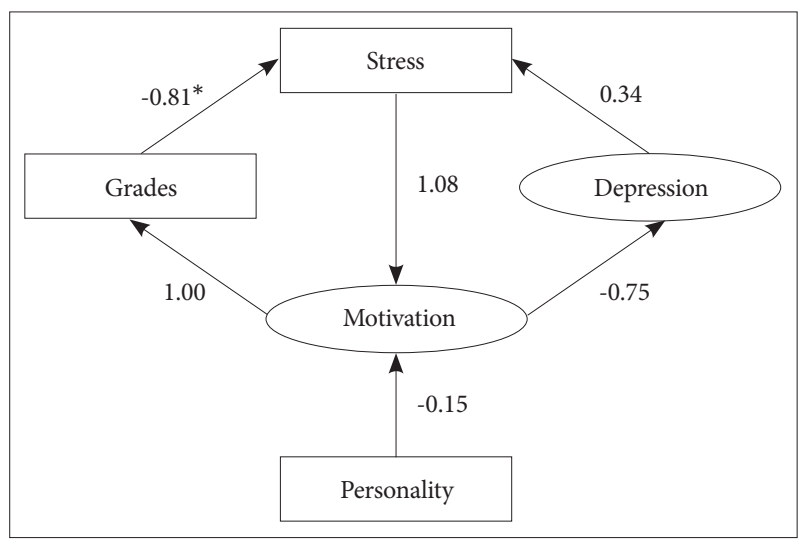

Figure 2. A path-analysis model of the relationship among academic stress, academic motivation, personality, and grades in medical students. $X^{2}=20.28, d f=20, p=0.44$, Comparative Fit Index $(\mathrm{CFI})=1.00$, Normed Fit Index $(\mathrm{NFI})=0.97$, Root Mean Square Error of Approximation (RMSEA) $<0.01$ Rectangles and ovals represent observed and unobserved variables, respectively. Numbers printed next to single-headed arrows correspond to standardized regression weights. ${ }^{*} p<0.01$. The Academic Motivation Scale, the Medical Stress Scale, and the Minnesota Multiphasic Personality Inventory (MMPI-2) were used to evaluate the corresponding variables in this model. Personality was defined as the number of MMPI clinical scales with a T-score above 65 . The following details were not included in this diagram: 1) all subscales of the Academic Motivation Scale were included as indicators of the unobserved variable motivation and were arranged as covariates of each other and of and personality and 2) the Hamilton Depression Scale (HAMD-17) and Beck Depression Inventory (BDI-II) results were arranged as indicators of the unobserved variable depression and were arranged as covariates of each other, of the observed variable personality, and of the Academic Motivation subscale amotivation. The data used in the present study were cross-sectional, and the associations implied by our path analysis model are hypothetical.

vealed that motivation, grades, and stress may be sequentially associated with one another, forming a feedback loop. These factors may also be associated with depression and personality.

One of the implications of the path analysis model is that stress may be associated with motivation, and motivation may have an indirect association with stress through academic performance. Amotivation is defined as the absence of intent or drive. ${ }^{9}$ Therefore, students with higher amotivation scores may find it more difficult to maintain strong academic performance, especially in a highly competitive environment. This may also result in higher levels of stress. Extrinsic identified regulation is a type of extrinsic motivation that is similar to intrinsic motivation. ${ }^{9}$ Students who scored high on this subscale may be highly influenced by both internal and external factors. Moreover, school grades may influence both factors and consequently increase stress levels.

Several inferences may be drawn from our path analysis model. First, academic stress, motivation, and grades form a feedback loop. Therefore, if one of the three factors deteriorates, the other two factors may be stabilized as a protective psychological mechanism. For example, a decrease in grades would cause an increase in stress, which in turn may cause motivation to in- 
crease. This, in turn, would cause grades to increase, thereby correcting the initial decrease in grades. In real-life situations, the responses to each step would certainly be influenced and modified by other factors, and this protective mechanism may not function easily. From a practical viewpoint, however, our results imply that actions that promote motivation may greatly benefit from interventions against stress or depression. Moreover, appropriate stress management may increase motivation. Interestingly, the proposed psychological mechanism strikingly resembles hormonal negative feedback loops. Moreover, contradictory associations were noted with depression. Depression showed comparatively weak positive associations with academic stress, which in turn was positively associated with motivation, making the net effect positive. Depression also showed a stronger negative association with motivation. Several unsuccessful path models were constructed, so our results are inconclusive at this time.

Previous studies have not investigated the associations among the three factors (i.e., academic performance, academic stress, and academic motivation) in medical students. Two reports, however, focused on the relationship between two of the three factors. Specifically, one study examined the influence of perceived stress factors on academic performance in dental students and concluded that the association was non-significant. $^{24}$ This is contrary to the path analysis results in the present study; this discrepancy may be due to the use of different measures and differences in the study groups. The proportion of students in each grade was similar to that in the present study; however, the participants in the previous study were unevenly distributed. Additionally, our results indicate that stress may not be directly associated with academic performance. A second study investigated the influence of personality on intrinsic academic motivation in medical students. ${ }^{13}$ The authors reported that various aspects of personality were associated with motivation. These results are consistent with the present study. Different assessments of personality and motivation were used in the previous study, thereby limiting direct comparisons.

Several previous studies have revealed that stressed students were more likely to be in their first year of medical school compared with non-stressed students. This is, however, controversial. ${ }^{1,3,6}$ These findings suggest that the early years of medical school are the most stressful and that the ideal time for interventions might be at the beginning of medical school.

The present study has several limitations. First, some of the assessment tools that were used, especially the MMPI- 2 clinical scales and the depression scales, are designed for pathological assessments. Most of results from these tests were within the normal range and may not be clinically meaningful. The present study was also unable to identify the aspects of personality that have the strongest association with other factors. The tools that were used to measure stress and motivation were designed for use in a normal population, and thus the overall influence was probably minor. Next, many of the assessments were based on self-reports. Nevertheless, the self-report forms used in this study have all been validated, and therefore, this probably had very little effect. Moreover, unauthorized absences, dropout rates, and clinically significant levels of depression were not included in the final analyses because the number of students falling into these categories was too small $(n \leq 10)$ and the results were not significant. Finally, the present study was a cross-sectional survey and not a longitudinal follow-up study. Thus, it is not possible to infer causal associations based on the results of our path analysis. Future longitudinal studies that focus on stress, motivation, and academic performance in medical students are warranted to confirm our results.

Taken together, the results of the present study indicate that academic stress in medical students may be influenced by an interaction among motivation, school grades, depression, and personality. Therefore, strategies to reduce academic pressures in medical students should take into consideration the importance of these factors. Increasing motivation may occur through interventions against stress and depression. Moreover, appropriate stress management may help students to become more motivated. Screening students once a year with self-report measures that evaluate stress, depression, and motivation may be helpful. This would identify students in need of individual counseling for stress management. Furthermore, as the number of students suffering from significant levels of stress is expected to be high, universal interventions may also be beneficial. Future studies are warranted regarding the interaction between motivation and depression.

\section{REFERENCES}

1. Compton MT, Carrera J, Frank E. Stress and depressive symptoms/ dysphoria among US medical students: results from a large, nationally representative survey. J Nerv Ment Dis 2008;196:891-897.

2. Dahlin M, Joneborg N, Runeson B. Stress and depression among medical students: a cross-sectional study. Med Educ 2005;39:594-604.

3. Goebert D, Thompson D, Takeshita J, Beach C, Bryson P, Ephgrave K, et al. Depressive symptoms in medical students and residents: a multischool study. Acad Med 2009;84:236-241.

4. Guthrie E, Black D, Bagalkote H, Shaw C, Campbell M, Creed F. Psychological stress and burnout in medical students: a five-year prospective longitudinal study. J R Soc Med 1998;91:237-243.

5. Levey RE. Sources of stress for residents and recommendations for programs to assist them. Acad Med 2001;76:142-150.

6. Paro HB, Morales NM, Silva CH, Rezende CH, Pinto RM, Morales RR, et al. Health-related quality of life of medical students. Med Educ 2010; 44:227-235.

7. Stewart SM, Lam TH, Betson CL, Wong CM, Wong AM. A prospective analysis of stress and academic performance in the first two years of medical school. Med Educ 1999;33:243-250.

8. Vitaliano PP, Maiuro RD, Mitchell E, Russo J. Percieved stress in medical school: resistors, persistors, adaptors and maladaptors. Soc Sci Med 1989;28:1321-1329. 
9. Fairchild AJ, Horst SJ, Finney SJ, Barron KE. Evaluating existing and new validity evidence for the Academic Motivation Scale. Contemp Educ Psychol 2005;30:331-358.

10. Ang RP, Huan VS. Relationship between academic stress and suicidal ideation: testing for depression as a mediator using multiple regression. Child Psychiatry Hum Dev 2006;37:133-143.

11. Doherty EM, Nugent E. Personality factors and medical training: a review of the literature. Med Educ 2011;45:132-140.

12. Lievens F, Coetsier P, De Fruyt F, De Maeseneer J. Medical students' personality characteristics and academic performance: a five-factor model perspective. Med Educ 2002;36:1050-1056.

13. Tanaka M, Mizuno K, Fukuda S, Tajima S, Watanabe Y. Personality traits associated with intrinsic academic motivation in medical students. Med Educ 2009;43:384-387.

14. Robbins SB, Oh IS, Le H, Button C. Intervention effects on college performance and retention as mediated by motivational, emotional, and social control factors: integrated meta-analytic path analyses. J Appl Psychol 2009;94:1163-1184.

15. Ahn D, Park G, Baek KJ, Chung SI. Academic motivation, academic stress and perceptions of academic performance in medical students. Korean J Med Educ 2007;19:59-71.

16. Vallerand RJ. The academic motivation scale: a measure of intrinsic, extrinsic, and amotivation in education. Educ Psychol Meas 1992;52:1003-
1017.

17. Butcher JN, Graham JR, Ben-Porath YS, Tellegen A, Dahlstrom WG, Kaemmer B. MMPI-2: Manual for Administration and Scoring, 2nd ed. Minneapolis, MN: The University of Minnesota Press; 2001.

18. Hamilton M. A rating scale for depression. J Neurol Neurosurg Psychiatry 1960;23:56-62.

19. Rhee MK, Lee YH, Park SH, Sohn CH, Chung YC, Hong SK. A standardization study of the Beck Depression Inventory (BDI): Korean version (K-BDI): reliability and factor analysis. Korean J Psychopharmacol 1995:77-95.

20. Kramer GP, Bernstein DA, Phares V. Introduction to Clinical Psychology, 7th Edition. Upper Saddle River, NJ: Prentice Hall; 2008.

21. Beck AT, Ward CH, Mendelson M, Mock J, Erbaugh J. An inventory for measuring depression. Arch Gen Psychiatry 1961;4:561-571.

22. Yi JS, Bae SO, Ahn YM, Park DB, Noh KS, Shin HK, et al. Validity and reliability of the Korean version of the Hamilton Depression Rating Scale (K-HDRS). J Korean Neuropsychiatr Assoc 2005;44:456-465.

23. Hooper D, Coughlan J, Mullen M. Structural equation modelling: guidelines for determining model fit. Electron J Bus Res Methods 2008; 6:53-60.

24. Sanders AE, Lushington K. Effect of perceived stress on student performance in dental school. J Dent Educ 2002;66:75-81. 